\title{
PA-083 PREVALENCE AND FACTORS ASSOCIATED WITH HYPOCHOLESTEROLAEMIA AMONG ADULTS WITH PULMONARY TB AT DIAGNOSIS AND DURING TB TREATMENT IN KAMPALA
}

John Mukisa, Ezekiel Mupere, William Worodria. Makerere University, Uganda

\subsection{6/bmjgh-2016-000260.115}

Background Hypocholesterolaemia is associated with altered immune function, possible delayed conversion at two months, and increased risk of mortality. However, lipid profiles are not done routinely for tuberculosis (TB) patients and there is paucity of data regarding the prevalence of hypocholesterolaemia and its associated factors among adult bacteriologicallyconfirmed pulmonary tuberculosis patients.

Methods This was a cross sectional study that consecutively enrolled 323 participants at diagnosis, 2, 5, 6 and 8 months of TB treatment, between February and April 2016. Physical examination and a structured questionnaire (administered by an interviewer) were used for data collection. Lipid profiles were determined from fasting blood samples from participants. Descriptive statistics were used to describe the patterns of dyslipidaemias and prevalence of hypocholesterolaemia. Log-binomial regression methods were used to determine the independent factors associated with hypocholesterolaemia.

Results Hypocholesterolaemia was identified in 140/323 (43.3\%, 37.9-48.8) of adults with pulmonary TB with a high prevalence among those at diagnosis, 51/91 (56.0\%, 45.8-66.3) but a lower prevalence among those who were at completion of treatment: $19 / 59$ (32.2\%, 20.9-44.3). On multivariate analysis, male gender (PR 1.57, 95\% CI: 1.16-2.06), diabetes (PR 1.37, 95\% CI: $1.05-1.78)$ and duration of anti TB treatment (1.12, 1.07-1.20) were associated with hypocholesterolaemia. There was no significant association between HIV infection status, 


\section{Abstracts}

presence of cavities on chest x-ray and hypocholesterolaemia at diagnosis and during anti-TB treatment in this study.

Conclusions The overall prevalence of hypocholesterolaemia among participants was high. Males with pulmonary tuberculosis are $60 \%$ more likely to develop hypocholesterolaemia. There is a need for further research on dyslipidaemias in TB patients and policy improvements regarding assessment of these lipids and nutritional management. 